\title{
The ongoing search for simplifying fractional flow reserve assessment: the role of contrast medium
}

\author{
Pio Cialdella, Domenico D’Amario, Antonio Maria Leone \\ Department of Cardiovascular Sciences, Fondazione Policlinico Universitario Agostino Gemelli, Roma, Italy
}

Adv Interv Cardiol 2016; 12, 3 (45): 197-199

DOI: 10.5114/aic.2016.61638

The current knowledge on the pathophysiology of coronary artery stenosis stems from the seminal studies by Lance Gould, who first described the pressure/flow/ resistance characteristics, defining the coronary flow reserve as the ratio between hyperaemic and basal flow [1]. From the beginning it was clear, indeed, that this technique had several major limitations, and therefore the concept of fractional flow reserve (FFR) was introduced. The FFR was defined as the ratio of two flows, calculated from two pressure values, obtained during maximal hyperaemia [2]. Consequently, the achievement of hyperaemia is the crucial prerequisite to assess FFR correctly. In this regard, the administration of intravenous (i.v.) adenosine is still considered the gold standard. Even so, the correct achievement of maximal hyperaemia has been acknowledged as one of the major challenges of this technique, leading to significant FFR underutilization worldwide [3].

Indeed, i.v. adenosine is perceived as a time-consuming, relatively expensive tool, relatively uncomfortable for the patient. In order to circumvent, at least partially, these limitations, the vast majority of interventional cardiologists prefers the intracoronary (i.c.) route of administration, even in highly skilled centres [4]. However, the most favourable dose of adenosine to be administered is still a matter of debate. Our group has previously demonstrated that only a high dose bolus of $600 \mu \mathrm{g}$ of i.c. adenosine has an effect on FFR comparable to the i.v. route, but this is achieved at a higher risk of atrioventricular (AV) block [5]. For this reason, we suggested to perform increasing boli of i.c. adenosine up to $600 \mu \mathrm{g}$, switching to the i.v. route in case of AV block. Recently, Adjedj et al. suggested that the best combination of hyperaemia and safety could be achieved by injecting $200 \mu \mathrm{g}$ of adenosine in the left coronary artery (LCA) and $100 \mu \mathrm{g}$ in the right [6]. However, these doses are still associated with a significant rate of AV block without reaching maximal hyperaemia. If adenosine still has some drawbacks, other potentially valuable vasodilator agents do not perform better [7].

For these reasons, adenosine-free pressure-derived indices were proposed over the last years. In $2010 \mathrm{Ma}$ mas et al. proposed the simple resting $\mathrm{Pd} / \mathrm{Pa}$ value to predict positivity of FFR. Pd/Pa was demonstrated to be significantly correlated with FFR and relatively accurate in predicting a positive FFR with an area under the curve (AUC) of 0.86 , and the authors concluded that for values lower than 0.87 and higher than 0.95, FFR measurement could be avoided [8]. However, more than $50 \%$ of lesions were in between these two values of $\mathrm{Pd} / \mathrm{Pa}$. In 2012 Sen presented a new adenosine independent index: the instantaneous wave free ratio (iFR) [9]. The theoretical principle of this new index derives from the main assumption of FFR. Indeed, flow is linearly correlated with pressure when the resistance is constant and minimal, and this is what we do inducing hyperaemia. Sen et al. theorized that during the cardiac cycle there is a short diastolic period in which, spontaneously, resistances are constant and minimal. The authors called it the wave free period and proposed this new index, the iFR, calculated as the ratio of distal and proximal pressures in this short period. In the ADVISE study Sen et al. demonstrated a very good correlation and agreement between iFR and FFR and a strong predictive capacity and reproducibility of iFR. Unfortunately, in the subsequent VERIFY study [10], Berry et al. challenged significantly the accuracy of iFR, showing a less convincing correlation and agreement of iFR with FFR, especially in real intermediate coronary artery stenoses, which represent the setting where functional assessment is clinically important. Moreover, they found that iFR assessed during hyperaemia was significantly lower than iFR at rest, and more importantly that while standard iFR predicted a positive FFR similarly to resting

\section{Corresponding author:}

Antonio Maria Leone MD PhD, Department of Cardiovascular Sciences, Fondazione Policlinico Universitario Agostino Gemelli, Largo Agostino Gemelli 8, 00168 Roma, Italy, e-mail: antoniomarialeone@gmail.com

Received: 19.06.2016, accepted: 19.06.2016. 
$\mathrm{Pd} / \mathrm{Pa}$, iFR during hyperaemia was significantly more accurate. Taking together all these data suggested that the resistance during the so-called wave free period can be constant but not so minimal, as previously suggested. These results were confirmed in the RESOLVE study [11], in which iFR and resting $\mathrm{Pd} / \mathrm{Pa}$ had a very similar behaviour, were closely correlated with each other, and, more importantly, had an identical ability in predicting FFR, with an AUC of about 0.80 , in a large number of lesions. Given the limited accuracy of iFR, Petraco et al. in the ADVISE registry [12] proposed for the first time iFR as a complementary tool to FFR for the functional evaluation of coronary artery stenosis. He defined two cut-offs of iFR (0.86 and 0.93) below and above which adenosine for FFR assessment could be avoided. However, using this approach, still $43 \%$ of lesions would have required adenosine infusion.

At this point, we needed an easier way to functionally assess with accuracy coronary artery stenoses, something that could be used with confidence by every interventionalist. Previous studies, including those by Gould et al. showed that radiographic contrast medium, routinely used during coronary angiography, and during FFR assessment to check the correct positioning of the pressure wire, is able to induce hyperaemia, although inferior to adenosine [1]. Thus, we hypothesized that the $\mathrm{Pd} / \mathrm{Pa}$ ratio registered by the pressure wire during sub-maximal reactive hyperaemia induced by i.c. injection of conventional non-ionic contrast medium (CMR or contrast FFR, CFFR) could be sufficient for the assessment of the physiological severity of stenosis in a large number of cases, avoiding the drawbacks of adenosine injection. With this aim we conducted the RINASCI study, investigating 104 intermediate coronary artery stenoses in 80 patients in whom we performed CFFR assessment with a single injection of $6 \mathrm{ml}$ of radiographic contrast medium [13]. After $30 \mathrm{~s}$ following the return of $\mathrm{Pd} / \mathrm{Pa}$ ratio to the baseline value, we performed FFR assessment using i.c. or i.v. adenosine. We found a very close correlation and a strong agreement between CFFR and FFR in Bland-Altman analysis, and, more importantly, receiver operating characteristics (ROC) curve analysis showed excellent accuracy of the cFFR cutoff of $\leq 0.83$ in predicting an FFR value $\leq 0.80$. Thus, we demonstrated that CFFR is very accurate in predicting FFR, representing a potentially valid surrogate, and helping to limit the use of adenosine to doubtful cases.

The results of our study were subsequently confirmed by other reports, and all these studies represented the background for the CONTRAST study, a large multicenter study comparing the potential of cFFR in predicting FFR vs. resting indexes (resting $\mathrm{Pd} / \mathrm{Pa}$, iFR) [14]. The results of the CONTRAST study showed the superiority of CFFR in comparison with resting $\mathrm{Pd} / \mathrm{Pa}$ and $\mathrm{iFR}$ for predicting FFR. Moreover, resting $\mathrm{Pd} / \mathrm{Pa}$ and iFR provided equivalent diagnostic accuracy, and the cFFR/FFR hybrid approach was demonstrated to be superior to iFR/FFR or $\mathrm{Pd} / \mathrm{Pa}$ / FFR hybrid approaches.
In the present issue of the journal another piece of evidence in favour of cFFR (also known as CMR, as in our previous RINASCI study) is presented [15]. The authors clearly confirmed previous reports also using manual injection of contrast medium, again supporting the wide applicability of this novel technique. They rightly report that large studies are needed to finally translate CFFR into clinical practice. Fortunately, this evidence does now exist: recently, we designed the Multi-center Evaluation of the Accuracy of the Contrast MEdium INduced $\mathrm{Pd} / \mathrm{Pa}$ RaTiO in Predicting FFR (MEMENTO-FFR) study in order to further elucidate the accuracy of cFFR in predicting FFR in a large real world series of lesions requiring functional assessment for clinical purposes. MEMENTO-FFR was a spontaneous, international, multicentre, non-randomized, collaborative, retrospective pooled analysis of the accuracy of cFFR in predicting FFR in patients with coronary artery disease in whom physiological lesion assessment was clinically indicated. We studied a total of 1026 lesions in 962 patients in 10 centres of Italy, Spain, Portugal and France, comparing the accuracy of cFFR (and of resting $\mathrm{Pd} / \mathrm{Pa}$ ) in predicting FFR. The results were presented in October 2015 at the TCT conference (Leone et al. Featured Clinical Research Session TCT 2015). We demonstrated that CFFR showed a higher $r$ coefficient and a lower spread of data, with a very strong agreement with FFR in Bland-Altman analysis. More importantly, using ROC curve analysis, cFFR strongly predicted FFR with an AUC of 0.95 , significantly better than resting $\mathrm{Pd} / \mathrm{Pa}$. Finally, the cut-off of $\leq 0.85$ of cFFR had an accuracy of $89 \%$ in identifying a correct FFR and, using a hybrid approach with CFFR/FFR, a significantly lower number of lesions required adenosine compared with resting $\mathrm{Pd} / \mathrm{Pa}$ ( $22 \%$ vs. $44 \%$ ). Practically this means that the interventionalist can choose between two approaches: if he/she wants to avoid completely the use of adenosine (using the cFFR $\leq 0.85$ cut-off), he/she has to accept (slightly) missing a correct FFR in about 1 case in 10, whereas he/she can achieve 100\% accuracy using a hybrid approach with CFFR/FFR, limiting use of adenosine to about 1 case in 5 .

In conclusion, cFFR is a very accurate index, and its intrinsic easiness, wide availability and safety may allow the use of adenosine to be limited to doubtful cases, with meaningful savings in time and costs. This could open the doors of the catheterization laboratory to second level tests of "advanced coronary physiology" (e.g. coronary flow reserve, hyperemic microvascular resistance, index of microvascular resistance, zero pressure) for the minority of cases with doubtful results after cFFR/ FFR assessment.

\section{Conflict of interest}

The authors declare no conflict of interest. 


\section{References}

1. Gould KL, Lipscomb K, Hamilton GW. Physiologic basis for assessing critical coronary stenosis. Instantaneous flow response and regional distribution during coronary hyperemia as measures of coronary flow reserve. Am J Cardiol 1974; 33: 87-94.

2. De Bruyne B, Sarma J. Fractional flow reserve: a review invasive imaging. Heart 2008; 94: 949-59.

3. Pijls NH, Tonino PA. The crux of maximum hyperemia: the last remaining barrier for routine use of fractional flow reserve. JACC Cardiovasc Interv 2011; 4: 1093-5.

4. Toth G, Hamilos M, Pyxaras S, et al. Evolving concepts of angiogram: fractional flow reserve discordances in 4000 coronary stenoses. Eur Heart J 2014; 35: 2831-8.

5. Leone AM, Porto I, De Caterina AR, et al. Maximal hyperemia in the assessment of fractional flow reserve: intracoronary adenosine versus intracoronary sodium nitroprusside versus intravenous adenosine: the NASCl (Nitroprussiato versus Adenosina nelle Stenosi Coronariche Intermedie) study. JACC Cardiovasc Interv 2012; 5: 402-8.

6. Adjedj J, Toth GG, Johnson NP, et al. Intracoronary adenosine: dose-response relationship with hyperemia. JACC Cardiovasc Interv 2015; 8: 1422-30

7. McGeoch RJ, Oldroyd KG. Pharmacological options for inducing maximal hyperaemia during studies of coronary physiology. Catheter Cardiovasc Interv 2008; 71: 198-204.

8. Mamas MA, Horner S, Welch E, et al. Resting Pd/Pa measured with intracoronary pressure wire strongly predicts fractional flow reserve. J Invasive Cardiol 2010; 22: 260-5.

9. Sen S, Escaned J, Malik IS, et al. Development and validation of a new adenosine-independent index of stenosis severity from coronary wave-intensity analysis: results of the ADVISE (ADenosine Vasodilator Independent Stenosis Evaluation) study. J Am Coll Cardiol 2012; 59: 1392-402.

10. Berry C, van 't Veer M, Witt N, et al. VERIFY (VERification of Instantaneous Wave-Free Ratio and Fractional Flow Reserve for the Assessment of Coronary Artery Stenosis Severity in EverydaY Practice): a multicenter study in consecutive patients. J Am Coll Cardiol 2013; 61: 1421-7.

11. Jeremias A, Maehara A, Généreux P, et al. Multicenter core laboratory comparison of the instantaneous wave-free ratio and resting $\mathrm{Pd} / \mathrm{Pa}$ with fractional flow reserve: the RESOLVE study. J Am Coll Cardiol 2014; 63: 1253-61.

12. Petraco R, Escaned J, Sen S, et al. Classification performance of instantaneous wave-free ratio (iFR) and fractional flow reserve in a clinical population of intermediate coronary stenoses: results of the ADVISE registry. Eurolntervention 2013; 9: 91-101.

13. Leone AM, Scalone G, De Maria GL, et al. Efficacy of contrast medium induced $\mathrm{Pd} / \mathrm{Pa}$ ratio in predicting functional significance of intermediate coronary artery stenosis assessed by fractional flow reserve: insights from the RINASCI study. Eurolntervention 2015; 11: 421-7.

14. Johnson NP, Jeremias A, Zimmermann FM, et al. Continuum of vasodilator stress from rest to contrast medium to adenosine hyperemia for fractional flow reserve (FFR) assessment. JACC Cardiovasc Interv 2016; 9: 757-67.

15. Topcu S, Tanboğa IH, Aksakal E, et al. Efficacy of coronary fractional flow reserve using contrast media compared to adenosine. Adv Interv Cardiol 2016; 12: 212-6. 